\title{
DISPONIBILIDADE E FRACIONAMENTO DE CD, PB, CU E ZN EM FUNÇÃO DO PH E TEMPO DE INCUBAÇÃO COM O SOLO ${ }^{1}$
}

\author{
Availability and fractionation of $\mathrm{Cd}, \mathrm{Pb}, \mathrm{Cu}, \mathrm{AND} \mathrm{Zn}$ in soil as a function of \\ incubation time and $\mathrm{pH}$
}

\author{
Évio Eduardo Chaves de Melo², Clístenes Williams Araújo do Nascimentoº, \\ Ana Cristiane Queiroz Santos ${ }^{4}$, Anderson Santos da Silva ${ }^{5}$
}

\begin{abstract}
RESUMO
$\mathrm{O}$ pH e o tempo de contato influenciam a distribuição dos metais entre frações do solo e a eficiência da fitoextração. Objetivou-se, neste trabalho estudar a disponibilidade dos metais $\mathrm{Cd}, \mathrm{Pb}, \mathrm{Cu}$ e $\mathrm{Zn}$ para a fitoextração, bem como suas redistribuições no solo, em função do tempo de incubação em solo com e sem calagem. O solo recebeu $\mathrm{Cd}, \mathrm{Pb}, \mathrm{Cu}$ e $\mathrm{Zn}$ nas doses $20,150,100$ e 150 $\mathrm{mg} \mathrm{kg}^{-1}$, respectivamente, na forma de sal solúvel. As amostras foram incubadas por 210, 180,150, 120, 90, 60, 30 e 0,5 dia. Terminada a incubação, mucuna preta (Stizolobium aterrimum Piper \& Tracy) foi cultivada por 30 dias. EDTA $\left(10 \mathrm{mmol} \mathrm{kg}^{-1}\right)$ foi aplicado sete dias, antes da coleta das plantas. As amostras de solo foram submetidas à extração química e fracionada. A concentração de metais pesados e a calagem afetaram a produção de matéria seca da parte aérea e da raiz. Em solos sem calagem, o aumento da solubilidade dos metais aumentou a fitoextração de $\mathrm{Cd}$ e Zn, mesmo sem aplicação do EDTA. A aplicação do EDTA ao solo com calagem mostrouse eficiente para a fitoextração de $\mathrm{Pb}$ e $\mathrm{Cu}$. A calagem reduziu os teores disponíveis de $\mathrm{Cd}, \mathrm{Pb}, \mathrm{Cu}$ e $\mathrm{Zn}$. A calagem provocou redução nos teores de $\mathrm{Cd}, \mathrm{Pb}, \mathrm{Cu}$ e $\mathrm{Zn}$ trocáveis e aumento nas frações matéria orgânica, óxidos de ferro amorfo e cristalino.
\end{abstract}

Termos para indexação: Fitoremediação, metais pesados, contaminação de solos.

\begin{abstract}
It is known that $\mathrm{pH}$ and incubation time influence the distribution of metals into soil fractions and therefore affect phytoextraction. Taking this in account, the aim of this work was to study the fractionation and availability of heavy metals for phytoextraction, as a function of incubation period in soils with or without liming. The soil samples were applied to $\mathrm{Cd}, \mathrm{Pb}, \mathrm{Cu}$, and $\mathrm{Zn}$ at concentrations of $20,150,100$, and $150 \mathrm{mg} \mathrm{kg}^{-1}$, respectively, in the form of soluble salt. The samples were kept incubated for high incubation periods: $210,180,150,120,90,60,30$, and 0,5 day. After that, velvetbean (Stizolobium aterrimum Piper \& Tracy) was cultivated for 30 days. EDTA at $10 \mathrm{mmol} \mathrm{kg}^{-1}$ rate was applied 7 days before plant harvest. Soils samples were fractionated and extracted for the metals with $\mathrm{CaCl}_{2}$ solution. The concentration of heavy metals in soil and liming affected the dry matter production of roots and shoots. For non limed samples, the metals solubility increasing hastened the phytoextraction of $\mathrm{Cd}$ and $\mathrm{Zn}$, even without EDTA addition. EDTA application to limed samples was effective for $\mathrm{Pb}$ and $\mathrm{Cd}$ phytoextraction. The liming reduced the available concentrations of $\mathrm{Cd}, \mathrm{Pb}, \mathrm{Cu}$, and $\mathrm{Zn}$ as well as decreased the exchangeable contents of such metals. Liming also increased the $\mathrm{Cd}, \mathrm{Pb}$, $\mathrm{Cu}$, and $\mathrm{Zn}$ concentrations in the organic matter and oxides fractions.
\end{abstract}

Index terms: Phytoremediation, heavy metals, soil contamination.

(Recebido em 21 de setembro de 2006 e aprovado em 29 de agosto de 2007)

\section{INTRODUÇÃO}

Os metais vêm se acumulando em solos brasileiros, em razão de processos naturais, como deposições atmosféricas, ou antropogênicos via atividades industriais, disposição de lodo de esgoto, disposição de rejeitos ou subprodutos domésticos e industriais, utilização de fertilizantes e agrotóxicos. A contaminação do solo por metais pesados pode resultar em diminuição da atividade microbiana, da biodiversidade e da fertilidade, perdas de rendimento das colheitas e danos à saúde de seres humanos e animais pela contaminação da cadeia trófica (MCLAUGHLIN \& SINGH, 1999).

Numerosos esforços foram empreendidos no desenvolvimento de tecnologias para remediação de solos

\footnotetext{
1Parte da dissertação de mestrado do primeiro autor, apresentada ao Programa de Pós-Graduação em Ciência do Solo, Universidade Federal Rural de Pernambuco/UFRPE

2Doutorando em Ciência do Solo - Departamento de Ciência do Solo/DCS - Universidade Federal Lavras/UFLA - Cx. P. 3037 - $37200-000$ - Lavras, MG jetmelo@uol.com.br

3Doutor em Ciência do Solo, Professor, Pesquisador do CNPq - Departamento de Agronomia/DEPA - Universidade Federal Rural de Pernambuco/ UFRPE - Rua Dom Manuel de Medeiros, s/n - Dois Irmãos - 52171-900 - Recife, PE - clistenes@pq.cnpq.br

${ }^{4}$ Mestre em Ciência do Solo - Departamento de Agronomia/DEPA - Universidade Federal Rural de Pernambuco/UFRPE - Rua Dom Manuel de Medeiros, s/n - Dois Irmãos - 52171-900 - Recife, PE - acqs@uol.com.br

${ }^{5}$ Graduando em Agronomia - Departamento de Agronomia/DEPA - Universidade Federal Rural de Pernambuco/UFRPE - Rua Dom Manuel de Medeiros s/n - Dois Irmãos - 52171-900 - Recife, PE - bobsilva@hotmail.com
} 
contaminados, práticas ex situ, como métodos físicoquímicos de lavagem do solo, e práticas in situ, como imobilização de poluentes metálicos. Esses métodos de remediação são geralmente muito caros e, freqüentemente, prejudiciais para as propriedades do solo. A fitorremediação de solos contaminados por metais pesados tem atraído atenção pelo seu baixo custo e benefícios ambientais (SALT et al., 1998). Entre as técnicas mais eficientes de fitorremediação, a fitoextração consiste no uso de plantas para absorver, transportar e concentrar metais do solo para a parte aérea (NASCIMENTO \& XING, 2006). O emprego de agentes quelantes, como EDTA, HEDTA e ácido cítrico, podem aumentar a mobilidade dos metais no solo e suas acumulações em plantas (CHEN et al., 2003), aumentando, portanto, a eficiência da fitoextração.

A determinação da concentração de metais no solo e as formas químicas nas quais esses elementos se encontram ou predominam associados provêm de uma base racional para avaliar a sua disponibilidade e toxicidade potencial. $\mathrm{O}$ uso de técnicas de fracionamento permite deduções sobre a biodisponibilidade, mobilidade e transporte dos metais, dinâmica dos elementos-traços e transformações entre as diferentes formas químicas em solos poluídos (ARAÚJO \& NASCIMENTO, 2005). Todavia, há erros inerentes à técnica de fracionamento de metais atribuídos à literatura, que se referem às incertezas como: temperatura e tempo de secagem da amostra; tempo de contato de cada extrator com a amostra; volume do frasco extrator; tempo e método de agitação; temperatura ambiente; falta de seletividade de alguns extratores; e redistribuição (readsorção) dos metais entre as fases de extração (TESSIER et al., 1979). Apesar dos erros, tratam-se de valores relativos que são úteis na avaliação da dinâmica do metal no sistema solo-planta.

A aplicação de calcário em solo contaminado é utilizada para elevar o pH do solo e reduzir a concentração do metal em forma disponível mediante a precipitação desse com o íon carbonato. Essa reação pode reduzir a biodisponibilidade de metais pesados no solo e a absorção pela planta (CHLOPECKA \& ADRIANO, 1996). Outro fator a ser considerado quanto à retenção de metais pesados pelos colóides é seu tempo de reação com o solo. Segundo Barrow (1993), um maior tempo de contato propicia que o metal passe para formas mais estáveis, como óxidos cristalinos, uma vez que a reação pode continuar lentamente com a difusão dos íons adsorvidos para o interior das partículas, demonstrando a importância do tempo nas transformações do metal adicionado aos solos.

Objetivou-se, neste trabalho verificar a disponibilidade dos metais $\mathrm{Cd}, \mathrm{Pb}, \mathrm{Cu}$ e $\mathrm{Zn}$ para a fitoextração, bem como suas redistribuições no solo, em função do tempo de incubação dos metais em solo com e sem calagem.

\section{MATERIAL E MÉTODOS}

O solo utilizado no experimento foi classificado como Argissolo Vermelho-Amarelo distrófico. Amostras do solo terra fina seca ao ar (TFSA) foram utilizadas para a caracterização química e física (Quadro 1), conforme Embrapa (1999). O solo seco ao ar, destorroado e homogeneizado foi passado em peneira de $4 \mathrm{~mm}$ de abertura de malha. Para simular a contaminação com metais pesados, foram acrescentadas em sacos plásticos contendo $1 \mathrm{~kg}$ de solo, $\mathrm{CdCl}_{2}, \mathrm{PbCl}_{2}, \mathrm{CuSO}_{4} 5\left(\mathrm{H}_{2} \mathrm{O}\right)$ e $\mathrm{ZnCl}_{2}$, de modo a elevar a concentração de metais no solo para 20, 150, 100 e 150 $\mathrm{mg} \mathrm{kg}^{-1} \mathrm{de} \mathrm{Cd}, \mathrm{Pb}, \mathrm{Cu}$ e $\mathrm{Zn}$, respectivamente. Após a adição das soluções, as amostras de solo permaneceram incubadas durante $210,180,150,120,90,60,30$ e 0,5 dia mantidos à umidade em $80 \%$ da capacidade de campo.

Após o período de incubação, uma solução nutritiva foi aplicada para o fornecimento de nutrientes às plantas: 250, 240, 150 e $100 \mathrm{mg} \mathrm{kg}^{-1}$ de N, P, K e S, respectivamente, a partir de $\mathrm{NH}_{4} \mathrm{SO}_{2}, \mathrm{NH}_{4} \mathrm{H}_{2} \mathrm{PO}_{4}$ e $\mathrm{KNO}_{3}$; além dos micronutrientes $\mathrm{Fe}\left(\mathrm{FeSO}_{4} \cdot 7 \mathrm{H}_{2} \mathrm{O}\right), \mathrm{Mn}\left(\mathrm{MnCl}_{2} \cdot 4 \mathrm{H}_{2} \mathrm{O}\right), \mathrm{B}$ $\left(\mathrm{H}_{3} \mathrm{BO}_{3}\right)$ e $\mathrm{Mo}\left(\mathrm{Na}_{2} \mathrm{MoO}_{4} \cdot 2 \mathrm{H}_{2} \mathrm{O}\right)$ nas concentrações de 2,4 , 1 e $0,2 \mathrm{mg} \mathrm{kg}^{-1}$. A acidez, de metade das amostras, foi corrigida para $\mathrm{pH}$ na faixa de $6,5-7,0$, utilizando-se carbonato de cálcio e magnésio p.a. (na proporção molar de 3:1), em quantidades previamente definidas em ensaio de incubação, por um período de 45 dias. As amostras foram transferidas para vasos com capacidade de $1 \mathrm{~kg}$, nos quais foi semeado mucuna preta (Stizolobium aterrimum Piper \& Tracy), deixando-se, após o desbaste, duas plantas por vaso, as quais foram cultivadas por 30 dias. Sete dias antes da coleta do experimento foi aplicado um quelante sintético (EDTA), numa concentração de $10 \mathrm{mmol} \mathrm{kg}{ }^{-1}$.

Quadro 1 - Características químicas e físicas do solo utilizado no experimento.

\begin{tabular}{|lr|}
\hline \multicolumn{1}{|c|}{ Característica } & Valor \\
\hline $\mathrm{pH}($ água 1:2,5) & 4,3 \\
$\mathrm{CTC}\left(\mathrm{cmol}_{\mathrm{c}} \mathrm{dm}^{-3}\right)$ & 6,05 \\
$\mathrm{C} . \mathrm{O} .\left(\mathrm{g} \mathrm{kg}^{-1}\right)$ & 4,5 \\
$\mathrm{Fe}\left(\mathrm{mg} \mathrm{dm}^{-3}\right)$ & 99 \\
$\mathrm{Cu}\left(\mathrm{mg} \mathrm{dm}^{-3}\right)$ & $\mathrm{LD}$ \\
$\mathrm{Zn}\left(\mathrm{mg} \mathrm{dm}^{-3}\right)$ & 1,45 \\
$\mathrm{Mn}\left(\mathrm{mg} \mathrm{dm}^{-3}\right)$ & 1,48 \\
$\mathrm{Cd}\left(\mathrm{mg} \mathrm{dm}^{-3}\right)$ & $<\mathrm{LD}$ \\
$\mathrm{Pb}\left(\mathrm{mg} \mathrm{dm}^{-3}\right)$ & $\mathrm{LD}$ \\
Areia $\left(\mathrm{g} \mathrm{kg}^{-1}\right)$ & 566 \\
Silte $\left(\mathrm{g} \mathrm{kg}^{-1}\right)$ & 48 \\
Argila $\left(\mathrm{g} \mathrm{kg}^{-1}\right)$ & 386 \\
\hline
\end{tabular}

$<$ LD - abaixo do limite de detecção. 
As raízes foram separadas do solo e, juntamente com a parte aérea, foram secas em estufa, com circulação forçada de ar a $70^{\circ} \mathrm{C}$. Foram obtidas as massas da matéria seca da parte aérea e da raiz, as quais em seguida foram submetidas à digestão nitro-perclórica (EMBRAPA, 1999) para quantificação de metais pesados. Foi estimado ainda, o conteúdo desses elementos nas diferentes partes dos vegetais, assim como a remoção de metais: Remoção = PA x MS, onde PA = Concentração na parte aérea e MS = Produção de matéria seca.

Foram determinados nas amostras do solo, os teores disponíveis dos metais ( $\mathrm{Cd}, \mathrm{Pb}, \mathrm{Cu}$ e $\mathrm{Zn}$ ) por cloreto de cálcio a $10 \mathrm{mmol} \mathrm{L}^{-1}$ (NOVOZAMSKY et al., 1993). Esse extrator foi escolhido pela sua capacidade para extração de metais apenas da fase prontamente disponível. A extração seqüencial foi baseada no método de Shuman (1985), com exceção da fração óxido de ferro amorfo que foi obtida pelo método de Chao \& Zhou (1983). Esse fracionamento separa os metais nas frações trocável, ligados à matéria orgânica, óxido de ferro amorfo e óxido de ferro cristalino.

$\mathrm{O}$ ensaio foi montado em um delineamento de blocos casualizados em esquema fatorial, utilizando-se oito tempos de incubação de metais com o solo, em amostras com e sem calagem, com 3 repetições. Os dados obtidos foram submetidos à análise de variância e regressão. Como não foi possível um ajuste significativo dos dados aos diferentes modelos de regressão, optou-se pela aplicação do teste de média Scott-Knott, a 5\% de probabilidade.

\section{RESULTADOS E DISCUSSÃO}

O tempo de incubação dos metais com o solo e a aplicação de calagem influenciaram a produção de matéria seca da parte aérea e da raiz da mucuna preta (Quadro 2). A produção de matéria seca foi significativamente menor nos períodos de incubação (30 e 0,5 dia), quando comparado com 210 dias nas amostras com calagem.

A calagem reduziu significativamente os teores de metais disponíveis extraídos por $\mathrm{CaCl}_{2}$ no solo (Quadro 3). Em média, os teores de $\mathrm{Cd}, \mathrm{Pb}, \mathrm{Cu}$ e $\mathrm{Zn}$ decresceram 32, 100,97 e $63 \%$, respectivamente. O efeito da calagem sobre a disponibilidade dos metais catiônicos se deve, principalmente, ao aumento da CTC do solo e a precipitação com carbonatos (ALLOWAY, 1990).

Quadro 2 - Matéria seca da parte aérea e raiz $\left(\mathrm{g}\right.$ pote $\left.{ }^{-1}\right)$ em mucuna preta, em solo contaminado, sob diferentes períodos de incubação.

\begin{tabular}{|c|c|c|c|c|c|c|c|c|}
\hline & 210 dias & 180 dias & 150 dias & 120 dias & 90 dias & 60 dias & 30 dias & $0,5 \mathrm{dia}$ \\
\hline \multicolumn{9}{|c|}{ Sem Calagem } \\
\hline P. Aérea & $0,83 b$ & $0,82 b$ & $0,80 \mathrm{~b}$ & $0,86 \mathrm{~b}$ & $0,88 \mathrm{~b}$ & $1,16 \mathrm{a}$ & $0,73 b$ & $0,64 \mathrm{~b}$ \\
\hline Raiz & $0,24 b$ & $0,24 b$ & $0,19 b$ & $0,22 b$ & $0,22 b$ & $0,32 \mathrm{a}$ & $0,21 b$ & $0,15 \mathrm{c}$ \\
\hline \multicolumn{9}{|c|}{ Com Calagem } \\
\hline P. Aérea & $2,51 \mathrm{a}$ & $2,00 \mathrm{c}$ & $1,73 d$ & $1,97 \mathrm{c}$ & $2,19 b$ & $2,14 \mathrm{~b}$ & $1,93 \mathrm{c}$ & $1,69 d$ \\
\hline Raiz & $0,57 \mathrm{a}$ & $0,37 \mathrm{c}$ & $0,43 b$ & $0,41 b$ & $0,58 \mathrm{a}$ & $0,44 b$ & $0,44 b$ & $0,35 \mathrm{c}$ \\
\hline
\end{tabular}

Médias seguidas de letras iguais na linha não diferem estatisticamente (Teste Scott-Knott com $\mathrm{P}<0,05$ ).

Quadro 3 - Teores de metais extraído por $\mathrm{CaCl}_{2}\left(\mathrm{mg} \mathrm{dm}^{-3}\right)$ em solo contaminado com metais pesados, sob diferentes períodos de incubação.

\begin{tabular}{|lcccccccc|}
\hline & 210 dias & 180 dias & 150 dias & \multicolumn{7}{c}{120 dias } & 90 dias & 60 dias & 30 dias & 0,5 dia \\
\hline Cádmio & $10,2 \mathrm{e}$ & $10,9 \mathrm{~b}$ & $12,3 \mathrm{a}$ & $11,1 \mathrm{~b}$ & $10,6 \mathrm{~d}$ & $10,5 \mathrm{~d}$ & $10,8 \mathrm{c}$ & $10,8 \mathrm{c}$ \\
Chumbo & $19,2 \mathrm{c}$ & $20,1 \mathrm{c}$ & $23,7 \mathrm{~b}$ & $17,3 \mathrm{~d}$ & $15,5 \mathrm{e}$ & $16,7 \mathrm{~d}$ & $15,9 \mathrm{e}$ & $25,3 \mathrm{a}$ \\
Cobre & $15,2 \mathrm{c}$ & $16,3 \mathrm{c}$ & $19,4 \mathrm{~b}$ & $16,0 \mathrm{c}$ & $17,0 \mathrm{c}$ & $15,9 \mathrm{c}$ & $14,8 \mathrm{c}$ & $24,7 \mathrm{a}$ \\
Zinco & $59,3 \mathrm{f}$ & $69,6 \mathrm{~d}$ & $78,4 \mathrm{~b}$ & $73,1 \mathrm{c}$ & $63,8 \mathrm{e}$ & $59,6 \mathrm{f}$ & $58,8 \mathrm{f}$ & $81,8 \mathrm{a}$ \\
& & & & & & & \\
Cádmio & $6,3 \mathrm{f}$ & $7,1 \mathrm{~d}$ & $8,8 \mathrm{a}$ & $7,7 \mathrm{c}$ & $7,5 \mathrm{c}$ & $7,4 \mathrm{c}$ & $6,6 \mathrm{e}$ & $8,2 \mathrm{~b}$ \\
Chumbo & $0,00 \mathrm{a}$ & $0,00 \mathrm{a}$ & $0,00 \mathrm{a}$ & $0,00 \mathrm{a}$ & $0,00 \mathrm{a}$ & $0,00 \mathrm{a}$ & $0,00 \mathrm{a}$ & $0,00 \mathrm{a}$ \\
Cobre & $0,2 \mathrm{~d}$ & $0,3 \mathrm{c}$ & $0,6 \mathrm{~b}$ & $0,5 \mathrm{~b}$ & $0,3 \mathrm{c}$ & $0,8 \mathrm{a}$ & $0,4 \mathrm{c}$ & $0,9 \mathrm{a}$ \\
Zinco & $23,1 \mathrm{f}$ & $35,0 \mathrm{e}$ & $52,6 \mathrm{~b}$ & $47,5 \mathrm{c}$ & $42,9 \mathrm{~d}$ & $33,8 \mathrm{e}$ & $32,1 \mathrm{e}$ & $65,1 \mathrm{a}$ \\
\hline
\end{tabular}

Médias seguidas de letras iguais não diferem estatisticamente (Teste Scott-Knott, $\mathrm{P}<0,05$ ). 
O maior tempo de contato diminuiu significativamente os teores disponíveis dos metais pesados no solo (Quadro 3). A diferença entre 0,5 e 210 dias de incubação representou decréscimos de 13, 24, 40 e $39 \%$ nos teores disponíveis de $\mathrm{Cd}, \mathrm{Pb}, \mathrm{Cu}$ e $\mathrm{Zn}$, respectivamente. A menor redução para o $\mathrm{Cd}$ evidencia o alto potencial de mobilidade desse elemento, potencializado pelo fato de sua adsorção ao solo ser reduzida pelos outros metais $(\mathrm{Pb}, \mathrm{Cu}$ e $\mathrm{Zn})$ que apresentam maior afinidade pelos sítios de adsorção no solo.

Nos solos sem calagem, o Cd foi encontrado apenas nas frações matéria orgânica e, principalmente, na trocável; os teores nos óxidos de ferro ficaram abaixo do limite de detecção, indicando a baixa afinidade do elemento com ligações mais estáveis do solo (Quadro 4). Isso comprova a alta biodisponibilidade de $\mathrm{Cd}$, provavelmente pelo fato de sua ligação aos colóides do solo serem inibidas por outros íons metálicos, os quais possuem maior afinidade (HARTER, 1991). O tempo de incubação influenciou apenas o teor de Cd trocável, apresentando uma redução de $30 \%$ entre o $1^{\circ}$ (210 dias) e o $8^{\circ}$ tempo de incubação ( 0,5 dia).
Na presença da calagem, o Cd apresentou redução média de $60 \%$ na fração trocável, resultando em um aumento de $40 \%$ na fração ligada à matéria orgânica (Quadro 5). Esse resultado indica que a redução de Cd trocável foi resultado da imobilização desse elemento pela matéria orgânica. Segundo Alloway (1990), a adsorção/dessorção de $\mathrm{Cd}$ está relacionada principalmente ao $\mathrm{pH}$, em comparação com o $\mathrm{Cu}$ e $\mathrm{Pb}$. Nesse tratamento, o tempo de incubação não apresentou diferença significativa.

$\mathrm{O} \mathrm{Pb}$ no solo sem calagem apresentou-se ligado às frações trocável, matéria orgânica e óxido de ferro cristalino (Quadro 4). O tempo de incubação influenciou principalmente o teor de $\mathrm{Pb}$ na fração matéria orgânica, a qual apresentou redução de $33 \%$ entre 0,5 e 210 dias. A aplicação de calagem resultou, em média, em redução de $71 \%$ do $\mathrm{Pb}$ na fração trocável, provavelmente por causa da precipitação do metal pela elevação do $\mathrm{pH}$, e aumento de $40 \%$ na fração óxido de ferro cristalino, respectivamente (Quadro 5). O aumento do tempo de contato promoveu aumento de 61, 4, 32 e $21 \%$ de $\mathrm{Pb}$ nas frações trocável, matéria orgânica, óxidos de ferro amorfo e cristalino, respectivamente.

Quadro 4 - Concentração de metal nas frações do solo $\left(\mathrm{mg} \mathrm{dm}^{-3}\right)$ sem calagem: trocável (Tr), matéria orgânica (MO), óxido de ferro amorfo (OxFeA) e óxido de ferro cristalino $(\mathrm{OxFeC})$.

\begin{tabular}{|c|c|c|c|c|c|c|c|c|}
\hline & 210 dias & 180dias & 150 dias & 120 dias & 90dias & 60 dias & 30 dias & $0,5 \mathrm{dia}$ \\
\hline \multicolumn{9}{|c|}{ Sem Calagem } \\
\hline \multicolumn{9}{|l|}{ Cádmio } \\
\hline $\operatorname{Tr}$ & $7,3 \mathrm{a}$ & $6,5 b$ & $6,2 b$ & $6,5 b$ & $6,0 \mathrm{c}$ & $5,3 \mathrm{~d}$ & $6,9 a$ & $5,1 d$ \\
\hline MO & $3,0 \mathrm{~b}$ & $3,1 b$ & $3,1 b$ & $3,1 b$ & $3,3 a$ & $3,4 a$ & $3,2 b$ & $3,0 \mathrm{~b}$ \\
\hline $\mathrm{OxFeA}$ & $<\mathrm{DL}$ & $<\mathrm{DL}$ & $<\mathrm{DL}$ & $<\mathrm{DL}$ & $<\mathrm{DL}$ & $<\mathrm{DL}$ & $<\mathrm{DL}$ & $<\mathrm{DL}$ \\
\hline $\mathrm{OxFeC}$ & $<\mathrm{DL}$ & $<\mathrm{DL}$ & $<\mathrm{DL}$ & $<\mathrm{DL}$ & $<\mathrm{DL}$ & $<\mathrm{DL}$ & $<\mathrm{DL}$ & $<\mathrm{DL}$ \\
\hline \multicolumn{9}{|l|}{ Chumbo } \\
\hline $\operatorname{Tr}$ & $51,1 \mathrm{~b}$ & $50,3 b$ & $53,4 \mathrm{a}$ & $48,7 \mathrm{c}$ & $44,6 \mathrm{e}$ & $45,5 \mathrm{e}$ & $46,6 \mathrm{~d}$ & $51,5 b$ \\
\hline MO & $75,2 \mathrm{a}$ & $69,6 b$ & $75,5 \mathrm{a}$ & $66,0 \mathrm{c}$ & $62,0 \mathrm{~d}$ & $61,4 d$ & $66,1 \mathrm{c}$ & $50,7 \mathrm{e}$ \\
\hline $\mathrm{OxFeA}$ & $<\mathrm{DL}$ & $<\mathrm{DL}$ & $<\mathrm{DL}$ & $<\mathrm{DL}$ & $<\mathrm{DL}$ & $<\mathrm{DL}$ & $<\mathrm{DL}$ & $<\mathrm{DL}$ \\
\hline $\mathrm{OxFeC}$ & $0,4 \mathrm{~b}$ & $1,2 \mathrm{a}$ & $0,1 \mathrm{c}$ & $0,5 b$ & $0,1 \mathrm{c}$ & $0,8 \mathrm{a}$ & $0,1 \mathrm{c}$ & $1,0 \mathrm{a}$ \\
\hline \multicolumn{9}{|l|}{ Cobre } \\
\hline $\operatorname{Tr}$ & $17,5 \mathrm{e}$ & $19,4 d$ & $24,3 \mathrm{a}$ & $20,4 c$ & $20,5 \mathrm{c}$ & $19,2 d$ & $20,3 c$ & $21,4 b$ \\
\hline MO & $37,4 a$ & $37,7 \mathrm{a}$ & $37,9 \mathrm{a}$ & $37,4 a$ & $35,7 b$ & $35,0 \mathrm{~b}$ & $35,6 b$ & $27,1 \mathrm{c}$ \\
\hline $\mathrm{OxFeA}$ & $3,7 \mathrm{a}$ & $2,5 \mathrm{c}$ & $3,1 b$ & $2,6 \mathrm{c}$ & $2,4 \mathrm{c}$ & $1,7 \mathrm{~d}$ & $2,6 \mathrm{c}$ & $2,4 c$ \\
\hline $\mathrm{OxFeC}$ & $0,5 \mathrm{a}$ & $0,3 \mathrm{c}$ & $0,4 \mathrm{~b}$ & $0,3 \mathrm{c}$ & $0,3 \mathrm{c}$ & $0,3 c$ & $0,4 b$ & $0,3 c$ \\
\hline \multicolumn{9}{|l|}{ Zinco } \\
\hline $\operatorname{Tr}$ & $35,7 a$ & $31,7 b$ & $32,8 b$ & $39,2 \mathrm{a}$ & $33,2 b$ & $22,8 \mathrm{c}$ & $39,9 a$ & $44,9 \mathrm{a}$ \\
\hline MO & $6,3 a$ & $6,1 b$ & $6,5 \mathrm{a}$ & $6,1 b$ & $5,5 \mathrm{c}$ & $5,2 \mathrm{~d}$ & $6,0 \mathrm{~b}$ & $4,9 \mathrm{e}$ \\
\hline $\mathrm{OxFeA}$ & $0,1 \mathrm{a}$ & $0,1 \mathrm{a}$ & $0,1 \mathrm{a}$ & $0,1 \mathrm{a}$ & $0,1 \mathrm{a}$ & $0,1 \mathrm{a}$ & $0,1 \mathrm{a}$ & $0,1 \mathrm{a}$ \\
\hline $\mathrm{OxFeC}$ & $0,8 \mathrm{~b}$ & $1,0 \mathrm{a}$ & $0,9 \mathrm{~b}$ & $0,8 \mathrm{~b}$ & $1,2 \mathrm{a}$ & $1,2 \mathrm{a}$ & $0,8 \mathrm{~b}$ & $1,1 \mathrm{a}$ \\
\hline
\end{tabular}

Médias seguidas de letras iguais não diferem estatisticamente (Teste Scott-Knott, $\mathrm{P}<0,05)$. 
Quadro 5 - Concentração de metal nas frações do solo $\left(\mathrm{mg} \mathrm{dm}^{-3}\right)$ com calagem: trocável (Tr), matéria orgânica (MO), óxido de ferro amorfo (OxFeA) e óxido de ferro cristalino (OxFeC).

\begin{tabular}{|c|c|c|c|c|c|c|c|c|}
\hline & 210 dias & 180dias & 150 dias & 120 dias & 90dias & 60 dias & 30 dias & $0,5 \mathrm{dia}$ \\
\hline & \multicolumn{8}{|c|}{ Com Calagem } \\
\hline \multicolumn{9}{|l|}{ Cádmio } \\
\hline $\operatorname{Tr}$ & $2,5 \mathrm{c}$ & $2,6 b$ & $2,9 \mathrm{a}$ & $2,7 \mathrm{~b}$ & $2,4 c$ & $2,2 \mathrm{~d}$ & $2,6 b$ & $2,3 d$ \\
\hline MO & $5,7 \mathrm{a}$ & $5,2 \mathrm{~b}$ & $5,4 \mathrm{~b}$ & $5,1 \mathrm{~b}$ & $5,1 \mathrm{~b}$ & $5,0 \mathrm{~b}$ & $5,0 \mathrm{~b}$ & $4,7 \mathrm{c}$ \\
\hline OxFeA & $<\mathrm{DL}$ & $<\mathrm{DL}$ & $<\mathrm{DL}$ & $<\mathrm{DL}$ & $<\mathrm{DL}$ & $<\mathrm{DL}$ & $<\mathrm{DL}$ & $<\mathrm{DL}$ \\
\hline $\mathrm{OxFeC}$ & $<\mathrm{DL}$ & $<\mathrm{DL}$ & $<\mathrm{DL}$ & $<\mathrm{DL}$ & $<\mathrm{DL}$ & $<\mathrm{DL}$ & $<\mathrm{DL}$ & $<\mathrm{DL}$ \\
\hline \multicolumn{9}{|l|}{ Chumbo } \\
\hline $\operatorname{Tr}$ & $5,1 \mathrm{e}$ & $16,1 \mathrm{c}$ & $23,7 \mathrm{a}$ & $17,0 \mathrm{~b}$ & $13,1 \mathrm{~d}$ & $15,5 \mathrm{c}$ & $13,7 \mathrm{~d}$ & $13,0 \mathrm{~d}$ \\
\hline MO & $60,2 \mathrm{c}$ & $68,0 \mathrm{a}$ & $63,3 b$ & $62,5 b$ & $62,5 b$ & $63,2 b$ & $63,7 \mathrm{~b}$ & $62,4 \mathrm{~b}$ \\
\hline OxFeA & $1,1 \mathrm{c}$ & $1,1 \mathrm{c}$ & $0,6 \mathrm{~d}$ & $1,1 \mathrm{c}$ & $0,7 \mathrm{~d}$ & $2,3 \mathrm{a}$ & $1,3 \mathrm{c}$ & $1,6 \mathrm{~b}$ \\
\hline $\mathrm{OxFeC}$ & $0,5 b$ & $1,0 \mathrm{a}$ & $1,0 \mathrm{a}$ & $1,3 \mathrm{a}$ & $0,1 \mathrm{c}$ & $1,0 \mathrm{a}$ & $1,3 \mathrm{a}$ & $0,6 b$ \\
\hline \multicolumn{9}{|l|}{ Cobre } \\
\hline $\operatorname{Tr}$ & $2,1 \mathrm{c}$ & $4,2 b$ & $5,6 \mathrm{a}$ & $5,6 \mathrm{a}$ & $5,1 \mathrm{a}$ & $4,0 \mathrm{~b}$ & $5,0 \mathrm{a}$ & $5,6 \mathrm{a}$ \\
\hline MO & $66,0 \mathrm{e}$ & $75,8 \mathrm{~d}$ & $86,9 \mathrm{a}$ & $75,7 \mathrm{~d}$ & $83,4 \mathrm{~b}$ & $74,6 \mathrm{~d}$ & $80,0 \mathrm{c}$ & $76,4 d$ \\
\hline OxFeA & $5,5 \mathrm{a}$ & $7,0 \mathrm{a}$ & $4,4 b$ & $6,1 \mathrm{a}$ & $4,3 b$ & $5,2 \mathrm{~b}$ & $4,2 b$ & $6,2 \mathrm{a}$ \\
\hline $\mathrm{OxFeC}$ & $0,6 \mathrm{a}$ & $0,6 \mathrm{a}$ & $0,5 \mathrm{~b}$ & $0,6 \mathrm{a}$ & $0,5 \mathrm{~b}$ & $0,6 \mathrm{a}$ & $0,5 \mathrm{~b}$ & $0,6 \mathrm{a}$ \\
\hline \multicolumn{9}{|l|}{ Zinco } \\
\hline $\operatorname{Tr}$ & $0,6 \mathrm{c}$ & $7,1 \mathrm{a}$ & $3,9 b$ & $3,9 b$ & $1,7 \mathrm{c}$ & $1,7 \mathrm{c}$ & $2,8 \mathrm{~b}$ & $9,2 \mathrm{a}$ \\
\hline MO & $67,1 \mathrm{a}$ & $55,1 \mathrm{~b}$ & $46,5 \mathrm{c}$ & $48,1 \mathrm{c}$ & $49,7 \mathrm{c}$ & $50,9 c$ & $50,9 c$ & $49,1 \mathrm{c}$ \\
\hline OxFeA & $0,3 \mathrm{a}$ & $0,2 b$ & $0,2 b$ & $0,2 b$ & $0,2 b$ & $0,2 b$ & $0,2 b$ & $0,2 b$ \\
\hline $\mathrm{OxFeC}$ & $1,5 \mathrm{~b}$ & $1,5 \mathrm{~b}$ & $1,3 \mathrm{~b}$ & $1,3 \mathrm{~b}$ & $1,7 \mathrm{a}$ & $1,8 \mathrm{a}$ & $1,4 \mathrm{~b}$ & $1,4 \mathrm{~b}$ \\
\hline
\end{tabular}

Médias seguidas de letras iguais não diferem estatisticamente (Teste Scott-Knott, <0,05).

Os teores de $\mathrm{Cu}$ encontrados no solo sem calagem estão ligados principalmente às frações trocável e matéria orgânica (Quadro 4). No entanto, a concentração de $\mathrm{Cu}$ em forma trocável foi inferior ao teor do elemento na matéria orgânica, indicando a pouca afinidade dos sítios de troca catiônica pelo elemento (ATANASSOVA \& OKAZAKI, 1997; NASCIMENTO et al., 2003). Foi observado aumento de $18 \%$ da concentração de $\mathrm{Cu}$ na fração trocável e redução de 28,37 e $43 \%$ na fração matéria orgânica, óxidos de ferro amorfo e cristalino entre o maior e o menor tempo de incubação. A calagem promoveu decréscimo médio de $71 \%$ na fração trocável e aumento de 54,51 e $38 \%$ de Cu nas frações matéria orgânica, óxido de ferro amorfo e cristalino, respectivamente (Quadro 5), provavelmente por um processo de adsorção específica, resultando na diminuição na disponibilidade do elemento. No entanto, o maior tempo de contato com o solo provocou redução na concentração de $\mathrm{Cu}$ em todas as frações, principalmente na trocável $(63 \%$ entre 0,5 e 210 dias de incubação com o solo).

Nos solos sem calagem, o $\mathrm{Zn}$ foi retido principalmente em formas trocáveis e na matéria orgânica (Quadro 4), permanecendo lábil no período do experimento. Xiang et al. (1995) relataram que, em solos ácidos, a transformação da forma trocável para a fração orgânica pode ser importante para reduzir a toxicidade de $\mathrm{Zn}$ para as plantas. O tempo de incubação, nesse tratamento, influenciou principalmente a fração trocável, com redução de $21 \%$ aos 210 dias de contato com o solo. Após a calagem, o teor de $\mathrm{Zn}$ sofreu brusca redução média de $89 \%$ na fração trocável, com concomitante aumento de 89,47 e $34 \%$ na concentração do $\mathrm{Zn}$ retido pela matéria orgânica, óxidos de ferro amorfo e cristalino, respectivamente (Quadro 5). A matéria orgânica foi a principal responsável pela retenção de $\mathrm{Zn}$ nos solos submetidos à calagem. Segundo Isabelle \& Alian (2001), a aplicação de matéria orgânica em solos contaminados pode eficientemente reduzir a concentração de $\mathrm{Zn}$ na solução do solo. Xiang et al. (1995) reportaram aumentos nos conteúdos de Zn ligado à óxidos de ferro cristalinos e amorfos em solos calcários comparativamente a solos ácidos. O menor tempo de incubação provocou redução em $27 \%$ na concentração de $\mathrm{Zn}$ na matéria orgânica, quando comparado com o maior tempo de contato. 
Com exceção do $\mathrm{Pb}$, cuja taxa de recuperação foi de aproximadamente $85 \%$, é interessante observar a baixa recuperação dos metais (soma das frações) em relação ao total adicionado ao solo. Para $\mathrm{Cd}, \mathrm{Cu}$ e $\mathrm{Zn}$, esses valores foram de 50, 59 e 29\%, respectivamente. Uma parte desses metais foi evidentemente transferida para a fração residual (não determinada nesse trabalho), mas é possível que frações importantes para solos tropicais, tais como óxidos de alumínio, devam ser contempladas para melhor entendimento da distribuição dos metais no solo.
O EDTA mostrou-se eficiente para indução da fitoextração apenas do $\mathrm{Pb}$ e $\mathrm{Cu}$, visto que as concentrações na parte aérea da mucuna aumentaram em média 68 e $26 \%$, respectivamente, em relação ao solo sem calagem (Quadros 6 e 7).

Wu et al. (2004) relatam que a adição de $3 \mathrm{mmol} \mathrm{kg}^{-1}$ de EDTA aumentou as concentrações de $\mathrm{Cu}$ e $\mathrm{Pb}$ na parte aérea da Brassica juncea (L.) Coss. Segundo Jarvis \& Leung (2002) a adição de EDTA aumentou a translocação de $\mathrm{Pb}$ em Pinus radiata D. Don. Por causa da eficiência da translocação do quelante, as concentrações de $\mathrm{Pb}$ e $\mathrm{Cu}$, na biomassa radicular, reduziram 69 e $86 \%$, respectivamente.

Quadro 6 - Concentração de metal $\left(\mu \mathrm{g} \mathrm{kg}^{-1}\right)$ na parte aérea e raiz na mucuna preta, em solo contaminado sem calagem.

\begin{tabular}{|lrrrrrrrr|}
\hline & 210 dias & 180dias & 150 dias & 120 dias & 90dias & 60 dias & 30 dias & 0,5 dia \\
\hline Cádmio & & & \multicolumn{7}{c}{ Sem Calagem } \\
P. Aérea & $215,7 \mathrm{c}$ & $282,4 \mathrm{~b}$ & $290,9 \mathrm{~b}$ & $134,6 \mathrm{~d}$ & $141,2 \mathrm{~d}$ & $387,7 \mathrm{a}$ & $294,6 \mathrm{~b}$ & $191,3 \mathrm{c}$ \\
Raiz & $5853,6 \mathrm{a}$ & $4461,0 \mathrm{c}$ & $5231,7 \mathrm{~b}$ & $5050,1 \mathrm{~b}$ & $5067,1 \mathrm{~b}$ & $3305,9 \mathrm{e}$ & $5137,2 \mathrm{~b}$ & $3781,6 \mathrm{~d}$ \\
Chumbo & & & & & & & & \\
P. Aérea & $62,9 \mathrm{a}$ & $70,3 \mathrm{a}$ & $61,0 \mathrm{a}$ & $38,9 \mathrm{~b}$ & $52,65 \mathrm{~b}$ & $76,2 \mathrm{a}$ & $54,2 \mathrm{~b}$ & $75,1 \mathrm{a}$ \\
Raiz & $1368,7 \mathrm{c}$ & $1713,3 \mathrm{~b}$ & $3403,3 \mathrm{a}$ & $1282,5 \mathrm{c}$ & $1406,3 \mathrm{c}$ & $1883,2 \mathrm{~b}$ & $1749,1 \mathrm{~b}$ & $1202,4 \mathrm{c}$ \\
Cobre & & & & & & & & \\
P. Aérea & $58,9 \mathrm{~b}$ & $132,6 \mathrm{a}$ & $125,3 \mathrm{a}$ & $59,3 \mathrm{~b}$ & $74,6 \mathrm{~b}$ & $134,8 \mathrm{a}$ & $63,8 \mathrm{~b}$ & $83,4 \mathrm{~b}$ \\
Raiz & $6117,9 \mathrm{~d}$ & $6316,4 \mathrm{~d}$ & $9422,7 \mathrm{a}$ & $8476,4 \mathrm{~b}$ & $7298,6 \mathrm{c}$ & $5247,6 \mathrm{e}$ & $6461,9 \mathrm{~d}$ & $9412,2 \mathrm{a}$ \\
Zinco & & & & & & & \\
P. Aérea & $1532,6 \mathrm{~d}$ & $2006,2 \mathrm{~b}$ & $2941,3 \mathrm{a}$ & $1459,6 \mathrm{~d}$ & $1764,0 \mathrm{c}$ & $2241,9 \mathrm{~b}$ & $2135,6 \mathrm{~b}$ & $1445,7 \mathrm{~d}$ \\
Raiz & $15768,2 \mathrm{a}$ & $12148,8 \mathrm{c}$ & $13049,5 \mathrm{~b}$ & $15031,2 \mathrm{a}$ & $15454,0 \mathrm{a}$ & $10937,1 \mathrm{~d}$ & $13449,6 \mathrm{~b}$ & $15373,2 \mathrm{a}$ \\
\hline
\end{tabular}

Médias seguidas de letras iguais não diferem estatisticamente (Teste Scott-Knott, $\mathrm{P}<0,05$ ).

Quadro 7 - Concentração de metal $\left(\mu \mathrm{g} \mathrm{kg}^{-1}\right)$ na parte aérea e raiz em mucuna preta, em solo contaminado com calagem.

\begin{tabular}{|lcccccccc|}
\hline & 210 dias & 180dias & 150 dias & 120 dias & 90dias & 60 dias & 30 dias & 0,5 dia \\
\hline Cádmio & & & \multicolumn{7}{c}{ Com Calagem } \\
P. Aérea & $127,6 \mathrm{a}$ & $123,3 \mathrm{a}$ & $99,8 \mathrm{~b}$ & $98,6 \mathrm{~b}$ & $68,2 \mathrm{~d}$ & $89,0 \mathrm{c}$ & $55,4 \mathrm{e}$ & $89,6 \mathrm{c}$ \\
Raiz & $233,9 \mathrm{f}$ & $472,5 \mathrm{~d}$ & $324,7 \mathrm{e}$ & $535,4 \mathrm{c}$ & $203,0 \mathrm{f}$ & $855,2 \mathrm{a}$ & $663,8 \mathrm{~b}$ & $347,5 \mathrm{e}$ \\
Chumbo & & & & & & & \\
P. Aérea & $103,1 \mathrm{~d}$ & $186,3 \mathrm{~b}$ & $197,8 \mathrm{~b}$ & $170,7 \mathrm{~b}$ & $143,3 \mathrm{c}$ & $203,5 \mathrm{~b}$ & $144,2 \mathrm{c}$ & $402,5 \mathrm{a}$ \\
Raiz & $493,6 \mathrm{~b}$ & $743,3 \mathrm{a}$ & $530,8 \mathrm{~b}$ & $514,9 \mathrm{~b}$ & $576,7 \mathrm{~b}$ & $527,9 \mathrm{~b}$ & $533,1 \mathrm{~b}$ & $399,5 \mathrm{c}$ \\
Cobre & & & & & & & \\
P. Aérea & $83,5 \mathrm{c}$ & $138,9 \mathrm{~b}$ & $163,5 \mathrm{a}$ & $111,3 \mathrm{c}$ & $97,2 \mathrm{c}$ & $131,0 \mathrm{~b}$ & $98,6 \mathrm{c}$ & $160,3 \mathrm{a}$ \\
Raiz & $877,3 \mathrm{c}$ & $1130,6 \mathrm{~b}$ & $1141,0 \mathrm{~b}$ & $1079,0 \mathrm{~b}$ & $877,7 \mathrm{c}$ & $879,4 \mathrm{c}$ & $1031,2 \mathrm{~b}$ & $1312,5 \mathrm{a}$ \\
Zinco & & & & & & & \\
P. Aérea & $759,4 \mathrm{~d}$ & $890,2 \mathrm{c}$ & $887,8 \mathrm{c}$ & $1009,0 \mathrm{~b}$ & $790,1 \mathrm{~d}$ & $910,5 \mathrm{c}$ & $657,6 \mathrm{c}$ & $1480,2 \mathrm{a}$ \\
Raiz & $789,5 \mathrm{e}$ & $1602,0 \mathrm{c}$ & $1510,1 \mathrm{c}$ & $1306,3 \mathrm{~d}$ & $2625,2 \mathrm{a}$ & $2307,7 \mathrm{~b}$ & $1788,7 \mathrm{c}$ & $1275,6 \mathrm{~d}$ \\
\hline
\end{tabular}

Médias seguidas de letras iguais não diferem estatisticamente (Teste Scott-Knott, $\mathrm{P}<0,05$ ). 
O EDTA não foi eficiente no aumento da concentração de Cd e Zn na parte aérea da mucuna (Quadros 6 e 7), quando comparado com o solo sem a adição do quelante. Isso pode ser entendido pelo fato dos metais $\mathrm{Zn}$ e Cd serem naturalmente muito móveis na planta. Desse modo, no solo sem calagem, as concentrações médias do Cd e Zn na parte aérea foram 61 e $52 \%$ maiores que no solo com calagem (Quadros 6 e 7). A concentração do $\mathrm{Cd}$ na raiz também foi influenciada pelo $\mathrm{pH}$, pois foi reduzida em $90 \%$ após a calagem.
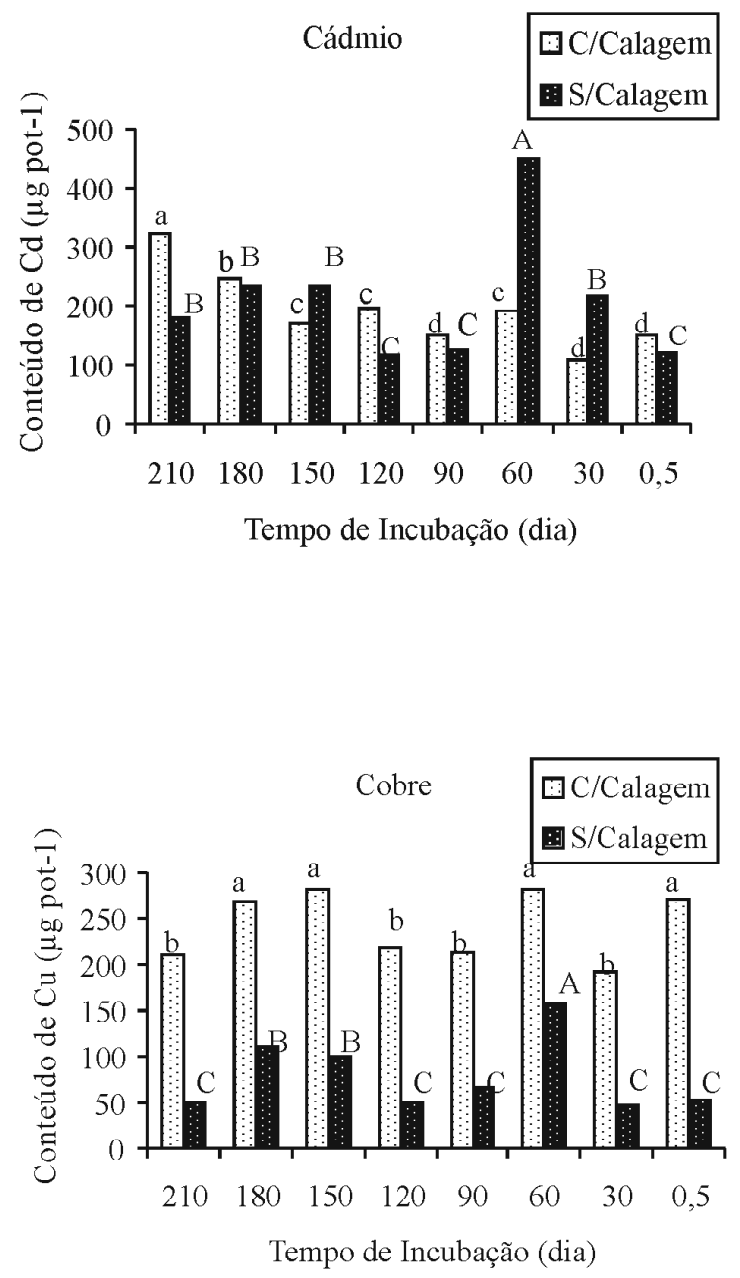

A remoção do Cd e Zn no solo sem calagem foram influenciadas principalmente pela acidez do solo (Figura 1), devido provavelmente à alta disponibilidade no solo desses elementos em pH baixo. A adição de EDTA ao solo com calagem representou pouca diferença para o $\mathrm{Cd}$ e o $\mathrm{Zn}$. Por outro lado, a remoção do $\mathrm{Pb}$ e $\mathrm{Cu}$ foi induzida pela adição desse quelante, pois esses elementos além da baixa mobilidade apresentam afinidade de formar em ligações mais estáveis no solo (ALLOWAY, 1990; MCBRIDE, 1994)
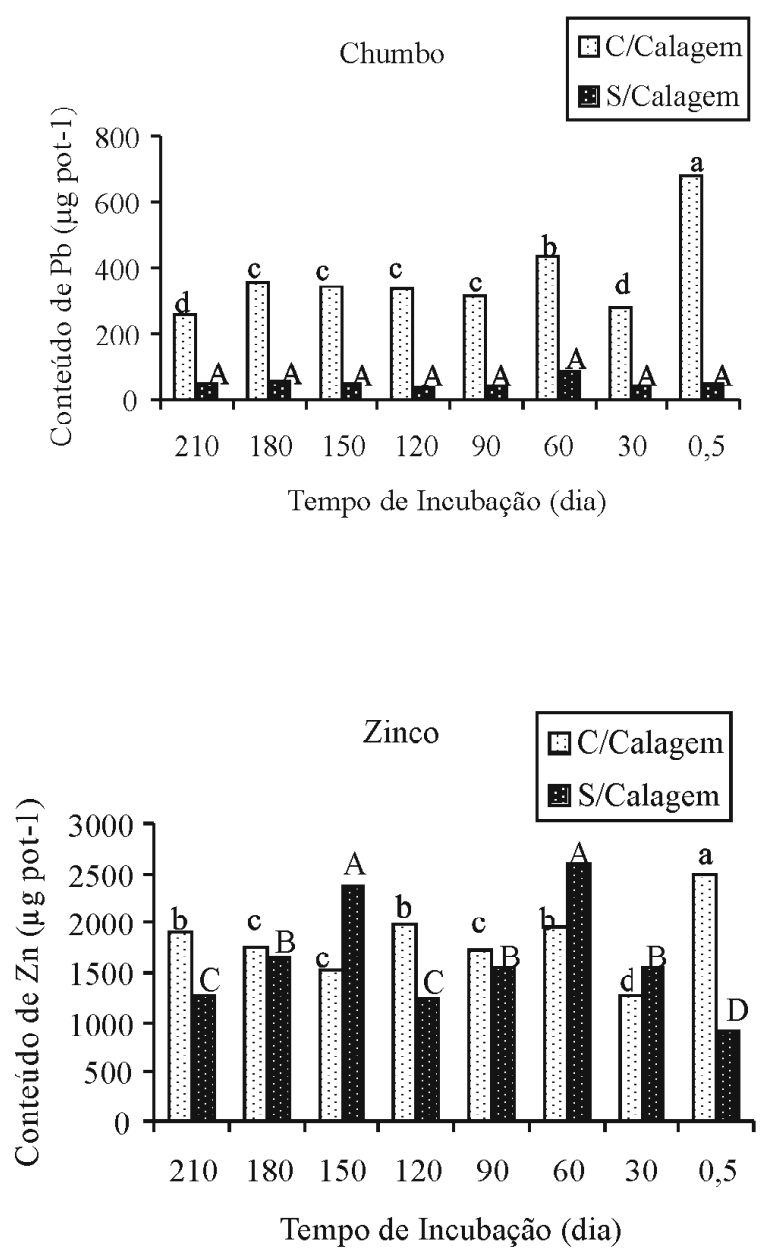

Figura 1 - Remoção de $\mathrm{Cd}, \mathrm{Pb}, \mathrm{Cu}$ e Zn pela parte aérea da mucuna preta $\left(\mu \mathrm{g}\right.$ pote $\left.{ }^{-1}\right)$ dos solos contaminados. Médias seguidas de letras iguais não diferem estatisticamente (Teste Scott-Knott com $\mathrm{P}<0,05$ ). 


\section{CONCLUSÕES}

A calagem reduziu significativamente os teores de $\mathrm{Cd}, \mathrm{Pb}, \mathrm{Cu}$ e $\mathrm{Zn}$ disponíveis extraídos por $\mathrm{CaCl}_{2}$ no solo e provocou redução nos teores de $\mathrm{Cd}, \mathrm{Pb}, \mathrm{Cu}$ e $\mathrm{Zn}$ trocáveis e aumento nas frações matéria orgânica, óxidos de ferro amorfo e cristalino.

Em solos sem calagem, o aumento da solubilidade dos metais influenciou a fitoextração de $\mathrm{Cd}$ e $\mathrm{Zn}$ pela mucuna preta sem a necessidade da aplicação de agente quelante (EDTA). A aplicação do EDTA no solo com calagem foi eficiente para a fitoextração induzida dos metais $\mathrm{Pb}$ e $\mathrm{Cu}$.

\section{REFERÊNCIAS BIBLIOGRÁFICAS}

ALLOWAY, B. J.Heavy metals in soils. New York: J. Wiley, 1990. 339 p.

ARAÚJO, J. C. T.; NASCIMENTO, C. W. A. Redistribuição entre as frações e teores disponíveis de zinco em solos incubados com lodo de esgoto. Revista Brasileira de Ciências do Solo, Campinas, v. 27, p. 451-459, 2005.

ATANASSOVA, I.; OKAZAKI, M. Adsorption-desorption characteristics of high levels of copper in soil clay fractons. Water, Air and Soil Pollution, [S.1.], v. 98, p. 213-228, 1997.

BARROW, N. J. Mechanisms of sorption of zinc in soil and soil components. In: Zinc in soils and plants. Dordrecht: Kluwer Academic, 1993. p. 15-31.

CHAO, T. T.; ZHOU, L. Extraction techniques for selective dissolution of amorphous iron oxides from soils and sediments. Soil Science Society American Journal, Madison, v. 47, p. 224-232, 1983.

CHEN, Y. X.; LIN, Q.; LUO, Y. M.; HE, Y. F.; ZHEN, S. J.; YU, Y. L.; TIAN, G. M.; WONG, M. H. The role of citric acid on the phytoremediation of heavy metal contaminated soil. Chemosphere, [S.1.], v. 50, p. 807-811, 2003.

CHLOPECKA, A.; ADRIANO, D. C. Mimicked in-situ stabilization of metals in a cropped soil: bioavailability and chemical form of zinc. Environment Science Technology, [S.l.], v. 30, p. 3294-3303, 1996.

EMPRESA BRASILEIRA DE PESQUISA AGROPECUÁRIA. Manual de análises químicas de solos, plantas e fertilizantes. Brasília, DF, 1999. 370 p.
HARTER, R. D. Micronutrient adsorption-desorption reactions in soils. In: MORTVERDT, J. J.; COX, F. R.; SHUMAN, L. M.; WELCH, R. M. Micronutrients in the agriculture. Madison: Soil Science Society American, 1991. p. $59-88$

ISABELLE, Y.; ALIAN, B. physical-chemical approach to assess the effectiveness of several amendments used for in situ remediation of trace metals-contaminated soils by adding solid phases. In: ISKANDER, I. K. (Ed.). Environmental restoration of metals-contaminated soils. Boca Raton: CRC, 2001. p. 3-20.

JARVIS, M. D.; LEUNG, D. W. M. Chelated lead transport in Pinus radiata: an ultrastructural study. Environmental and Experimental Botany, Oxford, v. 48, p. 21-32, 2002.

McBRIDE, M. B. Environmental chemistry of soil. New York: Oxford University, 1994. 406 p.

McLAUGHLIN, M. J.; SINGH, B. R. Cadmium in soils and plants: developments in plant and soil sciences. London: Kluwer Academic, 1999. 271 p.

NASCIMENTO, C. W. A.; FONTES, R. L. F.; MELÍCIO, A. Copper availability as related to soil copper fractions in oxisols under liming. Scientia Agricola, Piracicaba, v. 60, p. $167-173,2003$.

NASCIMENTO, C. W. A.; XING, B. Phytoextraction: a review on enhanced metal availability and plant accumulation. Scientia Agricola, Piracicaba, v. 63, n. 3, p. 299-311, 2006.

NOVOZAMSKY, I.; LEXMOND, T. M.; HOUBA, V. J. G. A single extraction procedure of soil for evaluation of uptake of some heavy metals by plants. International Journal of Environmental Analytical Chemistry, [S.1.], v. 51, p. 47-58, 1993.

SALT, D. E.; SMITH, R. D.; RASKIN, I. Phytoremediation. Annual Review Plant Physiology Plant Molecular Biology, [S.1.], v. 49, p. 643-668, 1998.

SHUMAN, L. M. Fractionation method for soil microelements. Soil Science, Baltimore, v. 140, p. 11-22, 1985.

TESSIER, A.; CAMPBELL, P. G. C.; BISSON, M. Sequential extraction procedure for the speciation of particulate trace metals. Analytica Chimica Acta, [S.1.], v. 51, p. 844-850, 1979. 
XIANG, H. F.; TANG, H. A.; YING, Q. H. Transformation and distribution of forms of zinc in acid, neutral and calcareous soils of China. Geoderma, [S.1.], v. 66, p. 121$135,1995$.
WU, L. H.; LUO, Y. M.; XING, X. R.; CHRISTIE, P. EDTA-enhanced phytoremediation of heavy metal contaminated soil with Indian mustard and associated potential leaching risk. Agriculture Ecosystems \& Environment, [S.1.], v. 102, p. 307-318, 2004. 